\title{
Minimal invasive distal pancreatectomy: Chronologic changes in the trends and clinical outcomes through an 15-year single center experience with 2,212 patients
}

Yejong PARK', Dae Wook HWANG', Jae Hoon LEE', Ki Byung SONG', Eunsung JUN ${ }^{1,2}$, Woohyung LEE', Jaewoo KWON', Song Cheol KIM"

'Division of Hepatobiliary and Pancreatic Surgery, Department of Surgery, Asan Medical Center, University of Ulsan College of Medicine, Seoul, Korea ${ }^{2}$ Department of Convergence Medicine, Asan Institute for Life Sciences, Asan Medical Center, University of Ulsan College of Medicine, Seoul, Korea

Introduction: Based on the development and expansion of treatment strategies since the 2000s, we identify the chronologic changes of the clinical and postoperative outcomes of minimal invasive distal pancreatectomy (MIDP) for left-sided pancreatic tumor.

Methods: Between 2005 and 2019, 2,212 patients underwent planned MIDP. The chronologic change in MIDP was analyzed according to procedure (open distal pancreatectomy [OPD] vs. MIDP), age, spleen preserving, and maligancy. In addition, the difference in clinical and postoperative outcomes was identified by dividing the periods at 5-year intervals, and prognostic factors of open converison and severe postoperative complication were analyzed.

Results: MIDP has been increasing steadily over 15 years when comparing ODP (from $10.8 \%$ to $84.9 \%$ ). Benign disease has a plateau pattern with a proportion of around $90 \%$ from 2010, but in pancreatic ductal adenocarcinoma (PDAC), the proportion of MIDP from $5.6 \%$ to $68.4 \%$ has been steadily increasing. In the second half of 15 years, compared to the first five years, PDAC increased from $8.3 \%$ to $32.8 \%$, and above all, the proportion of RAMPS increased significantly. The open conversion rate was $3.5 \%$, and the risk factors for open conversion in multivariate logistic regression analysis was malignancy, extended pancreatectomy, and site of tumor lesion (body). Also, the poor prognostic factor of severe complication was malignancy, extended pancreatectomy, and ASA classification $\geq 3$.

Conclusions: In benign disease, MIDP is considered a standard procedure. However, MIDP at PDAC still has a technical and oncological challenge. We need high quality randomized controlled trials for acceptable of MIDP in PDAC. 